\title{
The marine seismic vibrator: Designing a system for efficiency, improved data quality and reduced environmental footprint
}

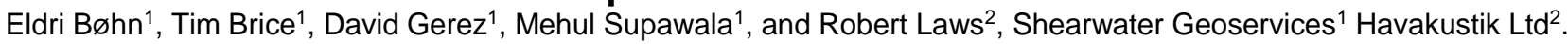

Copyright 2021, SBGf - Sociedade Brasileira de Geofísica

This paper was prepared for presentation during the $17^{\text {th }}$ International Congress of the Brazilian Geophysical Society held in Rio de Janeiro, Brazil, 16-19 August 2021.

Contents of this paper were reviewed by the Technical Committee of the $17^{\text {th }}$ International Congress of the Brazilian Geophysical Society and do not necessarily represent any position of the SBGf, its officers or members. Electronic reproduction or storage of any part of this paper for commercial purposes without the written consent of the Brazilian Geophysical Society is prohibited.

\section{Abstract}

A practical marine vibrator system has been designed and field tested with the aim of reducing the environmental impact and improving efficiency of marine seismic surveys. In order to achieve these goals, the vibrator units must meet an exacting set of standards and specifications. This has been achieved by the extensive use of modeling and simulation to provide insight and reduce risk during the development process.

Because the marine vibrator distributes energy over time, the instantaneous pressure is significantly lower than the peak output of an airgun array. More importantly, the SEL (Sound Exposure Level) criteria set a higher injury threshold for vibrators than for airguns. Even if it emits the same total energy, the vibrator is less damaging. The marine vibrator is therefore an environmentally friendly alternative to airguns

We also show how phase control, enabled by high-fidelity marine seismic vibrators, can be used to acquire and separate high multiplicity, simultaneous-source data. By changing the phase of the source wavefield from shot to shot and following a prescribed phase sequence, energy from selected sources can be moved into different parts of the frequency wavenumber spectrum in the commonreceiver domain. We use synthetic examples to demonstrate how this type of seismic acquisition, combined with wavefield reconstruction techniques, has the potential to provide a new method of high-multiplicity simultaneous-source acquisition and separation.

\section{Introduction}

Seismic vibrators have been used on land since the early days of seismic exploration, but their use in the marine environment has so far been limited. There are two primary drivers for the use of marine vibrators which are reduced environmental impact and the potential to improve survey efficiency.

Marine seismic vibrators emit their energy spread out in time, as opposed to airguns, which emit the energy in a single, high-intensity pulse. This 'soft output' gives the marine vibrator an environmental advantage even if the total acoustic energy emitted is the same (Southall et al.,
2007; JASCO, et al 2018). A second environmental advantage stems from our ability to tailor the energy spectrum of the vibrator source to the minimum needed to satisfy the imaging requirements (Laws et al., 2018a). Because the marine vibrator distributes energy over time, the instantaneous pressure is significantly lower than the peak output of an airgun array. More importantly, the Southall (2007, 2019) SEL (Sound Exposure Level) criteria set a higher injury threshold for vibrators than for airguns thus the marine vibrator has a smaller environmental footprint compared to airguns.

In addition to the environmental benefit, marine vibrators offer geophysical benefits. The phase of the emitted signal from a marine vibrator array can be varied from one sweep to the next in a 'phase-sequence'. Carefully designed phase-sequences can attenuate residual source noise and thereby allow extra sweeps to be inserted into the source line. The phase of the individual vibrators within the source array can also be used to modify source array directivity. If a seismic line is acquired using a sequence of different directivities, much more information is collected per kilometre than is the case in conventional acquisition (Laws and Halliday 2013).

For a marine vibrator to be suitable for these techniques it must, in general terms be a high-fidelity device. The precise device specifications can be defined by using realistic end-to-end simulations of the physical systems and of the data processing. The specifications are somewhat more onerous than for a conventional vibrator but are achievable.

In this abstract we describe the engineering and in-water testing of a marine vibrator system that can exploit these techniques while, at the same time, delivering enough energy to obtain a full seismic image.

\section{Development concept}

The marine-vibrator concept has been investigated for three to four decades, but it has not been developed for commercial use so far. A different approach has been taken here in that we have developed concepts to exploit the ability to control precisely the amplitude, frequency and phase of the output signal, something that airguns do not allow. Combining this with complementary data processing we can use phase encoding and multiple units to emit directional energy which have the potential to reduce survey duration. We have conducted specific field experiments to characterize the energy that is required to successfully image a reservoir.

The low frequency energy which is key for seismic imaging has been a challenge for past marine vibrator designs. One of the fundamental technical challenges is driving the transducer, as this requires an actuator 
exerting high forces over long displacements through the full 3-150 Hz bandwidth mandated by our requirements. By careful study of various vibrator designs we reached the conclusion that the optimal drive technology is electrohydraulic.

We can maximize the acoustic energy by matching the depth of each transducer to the frequency band of its sweeps, but the resulting multi-depth geometry results in mechanical challenges. Similarly, producing a directional signal by combining two transducers into a gradient source (Laws et al., 2018b) places stringent demands on the precision of the towing geometry. We developed finite-element models of potential hardware designs and apply them in simulations under realistic current and seastate conditions.

Modularity is an important aspect of the marine-vibrator system. The transducer tests and actuator trade-off study established limits on the acoustic output of each transducer, and the corresponding weights, dimensions, and power consumption. The results of the handling study further constrained the range of options to those that can be safely deployed while enabling high operational uptimes. These physical concerns, along with others, interact with geophysical goals. For example, the goal of maximizing the signal-to-noise ratio calls for powerful individual transducers, while the differences among operational scenarios call for dividing the acoustic output among several individual transducers emitting distinct signals from separate positions (Figure 1). To better understand these complex multi-dimensional trade-offs, we linked the results of different modelling domains. We concluded that distributing the output among several independently towed transducers is the optimal way to achieve our operational scenarios.

\section{Engineering development}

We developed and tested multiple generations of the transducer at the Seneca Lake Sonar Test Facility. We tested our third-generation transducer (Figure 1) and concluded that it has sufficient output - especially at low frequencies - to achieve our design goals and satisfy all operational scenarios we have set.

Having defined the operational requirements and derived system requirements, we are able to work on various decoupled sub-systems while respecting the required interfaces. Going forward, we will individually test each component, and then take on a bottom-up integration practice to incrementally integrate multiple components into subsystems of increasing scope and complexity. We will use previously derived models to predict the expected results, and we will seek to confirm our expectations in physical tests. The test results will in turn be used to calibrate our models and our proposed geophysical techniques, one of which is described below.

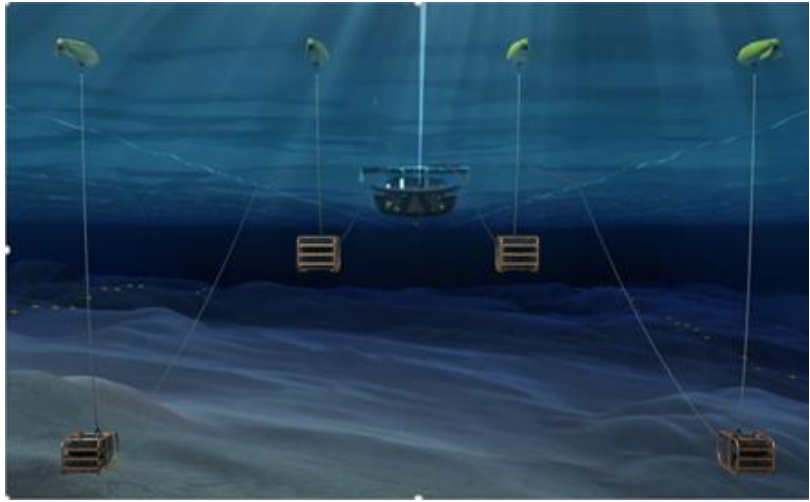

Figure 1 An example of a configuration with four projector modules towed behind the seismic vessel. The units are deployed at different depths.

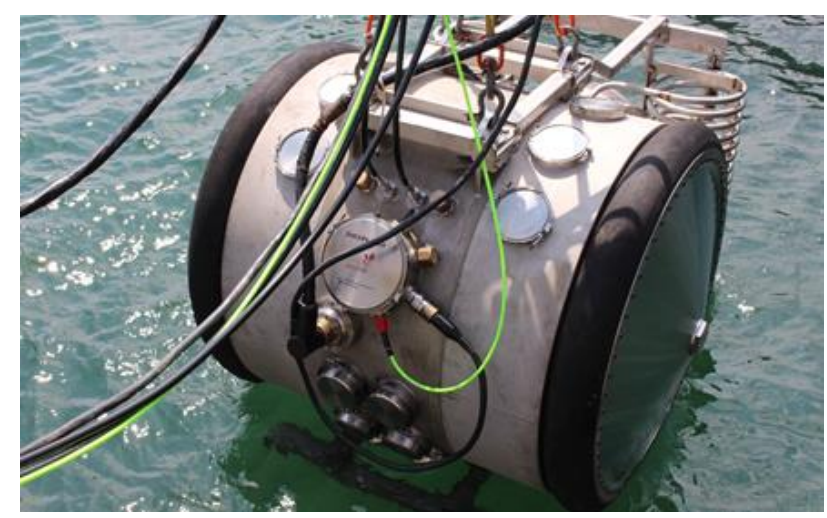

Figure 2 Transducer testing

\section{Phase sequencing of marine vibrators}

Phase sequencing refers to a method of marine seismic source encoding, in which the phase of the source wavefield is varied from shot-to-shot according to a predefined sequence of phases (Laws and Halliday 2013). The sequence of phases is chosen such that the source energy is shifted coherently in the frequencywavenumber $(f-k)$ domain of a common receiver gather (CRG). By using different phase sequences, the energy from different sources can be shifted to different parts of the $t-k$ domain, and wavefield reconstruction approaches can be used to separate the different source wavefields. This would enable the acquisition of a number of source lines simultaneously, thus improving survey efficiency. Other authors have proposed the use of time-delay sequences. However, a time delay changes the phase as a linear function of frequency, and the desired phase sequence can only be achieved for integer multiples of a particular frequency.

We demonstrate this encoding using a synthetic example modelled with 3D acoustic finite differences using the SEAM Phase 1 model. There is a single receiver on the seabed at a depth of $1900 \mathrm{~m}$, and source lines are modelled with a $15 \mathrm{~m}$ shot interval, with $30 \mathrm{~m}$ between 6 adjacent source lines. This gives an inline Nyquist wavenumber of 0.033 cycles $/ \mathrm{m}$. The source signature is flat in the bandwidth 2 to $60 \mathrm{~Hz}$. Source depth is $10 \mathrm{~m}$. The data for each of the six sources lines has a different phase sequence applied. The first source line has 
constant phase from shot to shot, the second increases in phase by $60^{\circ}$ from shot to shot, the third by $120^{\circ}$, the fourth by $180^{\circ}$, the fifth by $240^{\circ}$ and the sixth by $300^{\circ}$. The effect of this is to shift the entire contribution of each source along the wavenumber axis by $0,0.011,0.022$, $0.033,-0.022$ and -0.011 cycles $/ \mathrm{m}$, respectively. Windows of the CRG for each shot line are plotted in Figures $3 a$ to $3 \mathrm{f}$, with the summation of all six plotted in Figure $3 \mathrm{~g}$. The effect of the phase sequencing is to give a wavefield in the time-offset domain that does not look like seismic data. The $f-k$ spectra of the full gathers are plotted in Figures $3 \mathrm{~h}$ to $3 \mathrm{n}$, where the shifting of the signal cones becomes clear. In Figure $3 n$, we can see that the combined phase-sequenced data appear very similar to spatially aliased data, but in this case each of the signal cones corresponds to a different source, rather than aliased replicas of the same source.

Halliday et al. (2017) described how phase-sequenced sources such as those in Figure 3 can be separated using multi-frequency approaches, exploiting the coherency of seismic data across frequencies. The result of separating the data using this approach (in small overlapping time and space windows) is shown in Figure 4. Figures $4 a$ to $4 \mathrm{f}$ show the separated shot lines for each of sources 1 to 6 , and Figures $4 \mathrm{~g}$ to $4 \mathrm{l}$ show the separation error (the difference between the modelled non-simultaneous data, and the separated data). The quality of the separation result is measured as the signal-to-residual cross talk ratio. In the window from 4 to $5 \mathrm{~s}$ this ratio is $\mathrm{s}$ approximately $28 \mathrm{~dB}$ across all 6 shots, indicating a highquality separation result. Halliday et al. (2017a) studied the difference between time dithering and phase sequencing; they showed that phase-sequenced marine vibrators allows significantly higher-multiplicity simultaneous source acquisition without increased crosstalk.

\section{Conclusions}

The efficiency and data quality of a seismic survey can potentially be improved using marine vibrators in place of airguns. In addition, the marine vibrator also offers environmental benefits. This is because, under the Southall (2007) criteria, the marine vibrator is considered to be a 'continuous' source and therefore less intrusive to marine life. We have applied systematic processes to develop a new marine-vibrator system. We have integrated computer models across technical disciplines to ensure that the physical system supports the geophysical objectives, thereby reducing risk and accelerating development.

A high-fidelity marine seismic vibrator would allow the acquisition and separation of high multiplicity simultaneous-source data. This uses a method referred to as phase sequencing, which allows the energy from different sources to be coherently shifted to different parts of the $f-k$ domain. These data can then be separated using wavefield reconstruction techniques. This approach was demonstrated using synthetic examples, showing that six simultaneous sources can be separated to a high degree of accuracy.

We have modelled and tested the operation of key subsystems including the full-scale transducer and are currently developing the full system. The resulting modular, field-configurable system supports the full range of operational scenarios.

\section{Acknowledgments}

We acknowledge the contributions of Håkon Aune, Adrien Bialek, Anders Boeen, Robert Breivik, Robert DeLaCroix, Espen Gulbransen, David Halliday, Jon-Fredrik Hopperstad, David Gerez, Ed Kragh, Ola Larsen, Martin Laycock, Bruno Lecointre, Andreas Michaelides, Thomas Murray, Ali Özbek, Mark Ozimek, Ricardo Quintanilla, John Sallas, Tormod Tjaberg, Björn Ullbrand, Rik Wemmenhove, Benjamin Whiting, and Ken Wittlief.

We would also like to thank Equinor and the Research Council of Norway for the financial support that makes this work possible.

\section{References}

HALLIDAY, D.F., R.M. LAWS, J-F HOPPERSTAD and M. SUPAWALA, 2018, Exploiting phase control of a marine seismic vibrator for high-multiplicity simultaneous source acquisition: EAGE Marine Acquisition Workshop 2018, Extended Abstracts, doi: 10.3997/2214-4609.201802105.

HALLIDAY, D.F., LAWS, R.M., ÖZBEK, A. and HOPPERSTAD, J.F. 2017. Separating simultaneous sources using phase sequencing and reconstruction in marine seismic vibrators. 79th EAGE Conference and Exhibition 2017.

LAWS, R. and HALLIDAY, D. 2013. Seismic acquisition using phase-shifted sweeps. US Patent publication number US20140278119.

LAWS, R.M., J. HOPPERSTAD, E. Kragh and D. HALLIDAY, 2018a, How can we emit enough energy from a marine seismic vibrator? 80th EAGE Conference and Exhibition, Extended Abstracts, doi: 10.3997/22144609.201800997.

LAWS, R.M., D. HALLIDAY, J.-F. HOPPERSTAD, D. GEREZ, M. SUPAWALA, A. ÖZBEK, T. MURRAY, E. KRAGH, 2018b, Marine vibrators: the new phase of seismic exploration: Geophysical Prospecting, doi: 10.1111/1365-2478.12708.

JASCO, LGL and BRUNE R, 2018 Determining the environmental impact of marine vibrator technology. The E\&P sound \& marine life joint industry programme report. https://gisserver.intertek.com/JIP/DMS/ProjectReports/Ca t1/JIP-Proj1.6.2_Mar\%20Vib\%20EA\%20Phase\%201\%20$\% 20$ Executive\%20Summary.pdf

SOUTHALL, B.L., A.E. BOWLES, W.T. Ellison, J.J. FINNERAN, R.L. GENTRY, C.R. GREENE, D. KASTAK, D.R. KETTEN, J.H. MILLER, P.E. NACHTIGALL, W.J. RICHARDSON, J.A. THOMAS, and P.L. TYACK, 2007, Marine Mammal Noise Exposure Criteria: Initial Scientific Recommendations, Aquatic Mammals, 33, 411-522.

SOUTHALL, B.L, J.J. FINNERAN, C. REICHMUTH, P.E. NACHTIGALL, D.R. KETTEN, A.E. BOWLES, W.T. ELLISON, D.P. NOWACEK, and P.L. TYACK, 2019, Marine Mammal Noise Exposure Criteria: Updated Scientific Recommendations for Residual Hearing Effects, Aquatic Mammals, 45, 125-232. 
a.

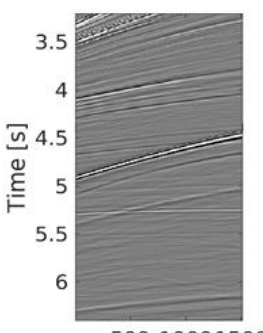

50010001500

h.

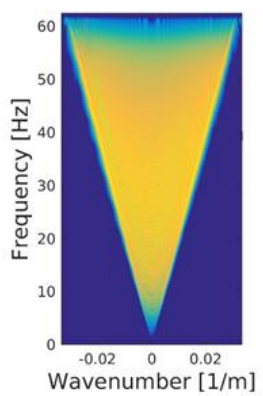

b.

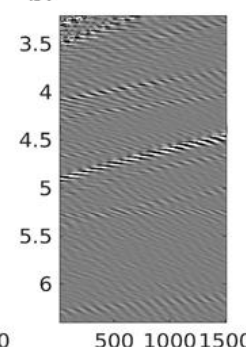

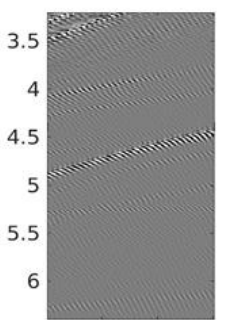

50010001500 d.

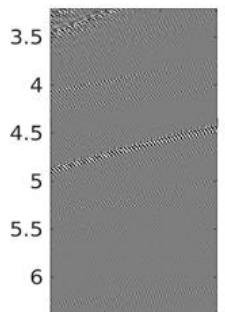

50010001500 e.

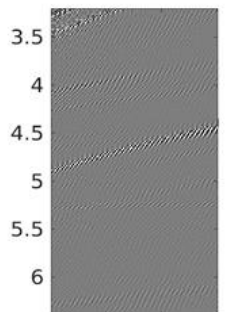

50010001500

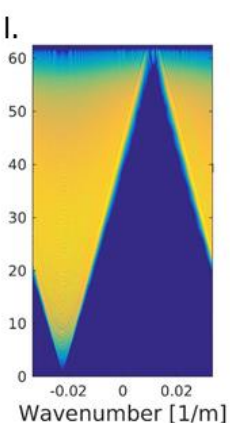

f.

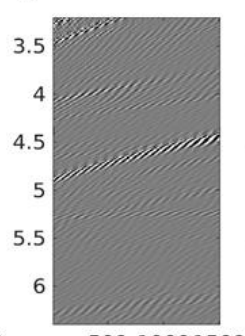

g.

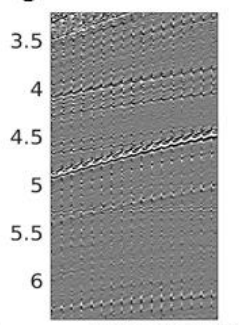

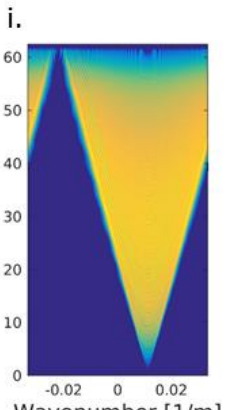

Wavenumber $[1 / \mathrm{m}]$
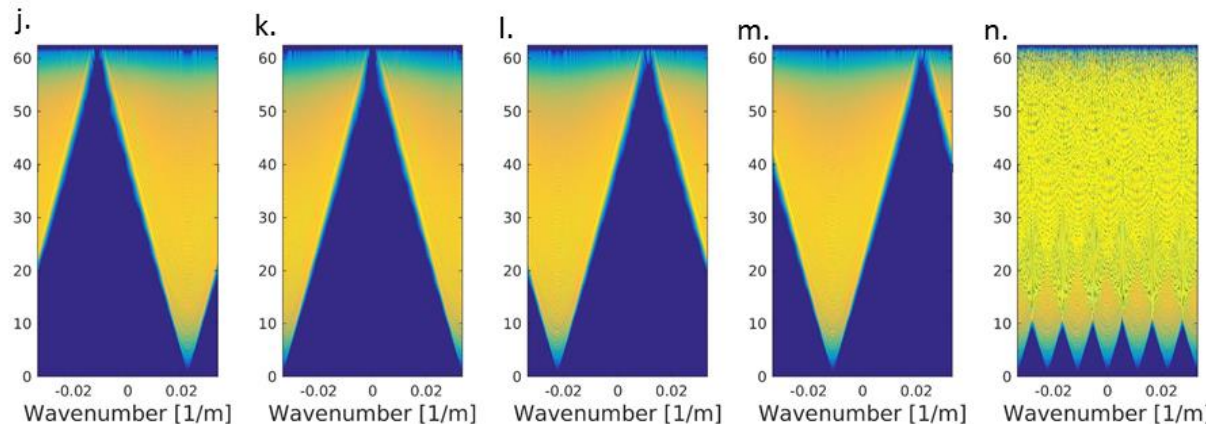

Figure 3 Encoding of six simultaneous sources using phase sequencing. (a) to (f) show six windows of data from six separate source lines with a shot-to-shot phase increment of $0^{\circ}, 60^{\circ}, 120^{\circ}, 180^{\circ}, 240^{\circ}$ and $300^{\circ}$, respectively. (g) Simultaneous source data produced by summing the data in $(\mathrm{a})$ to $(\mathrm{f})$. (h) to $(\mathrm{n})$, as for $(\mathrm{a})$ to $(\mathrm{g})$, but in the frequency-wavenumber domain

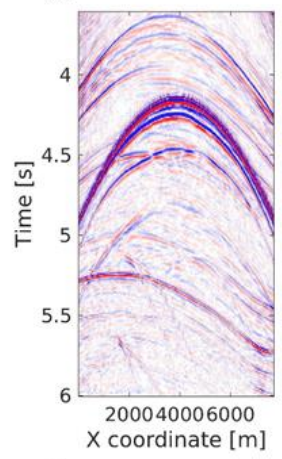

g.

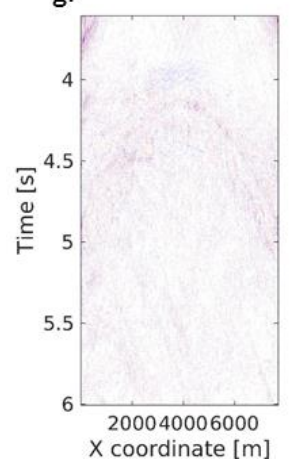

b.

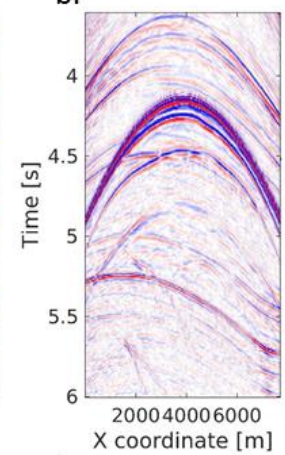

h.

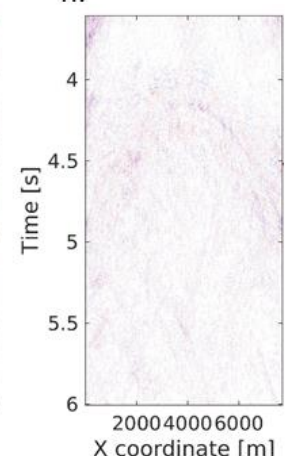

c.

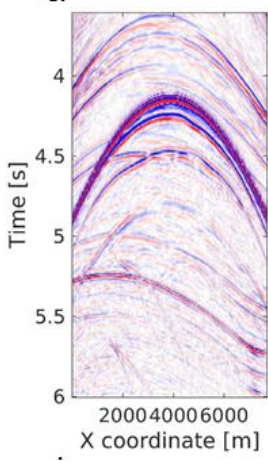

i.

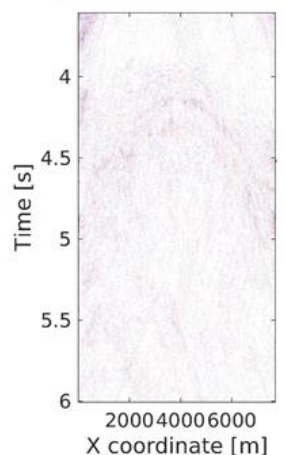

d.

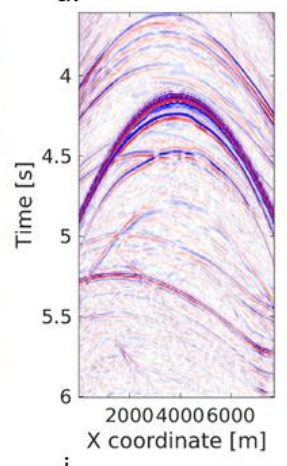

j.

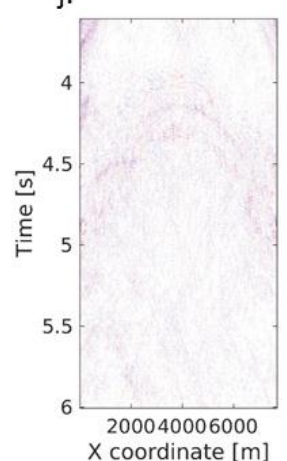

e.

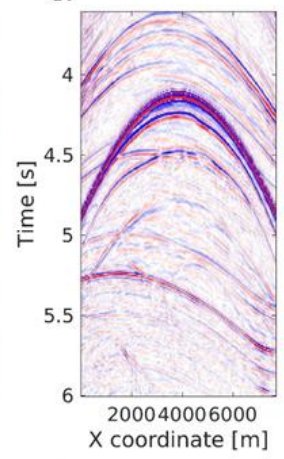

k.

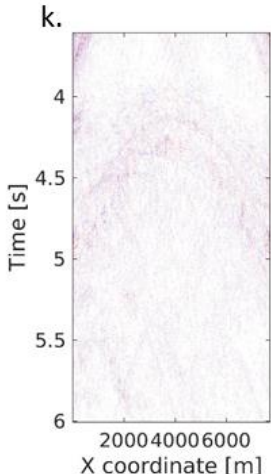

f.

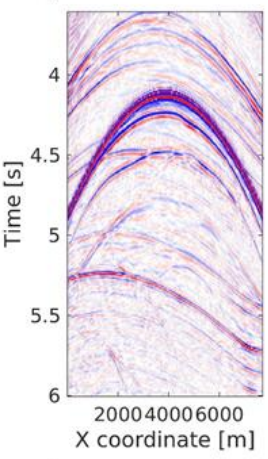

I.

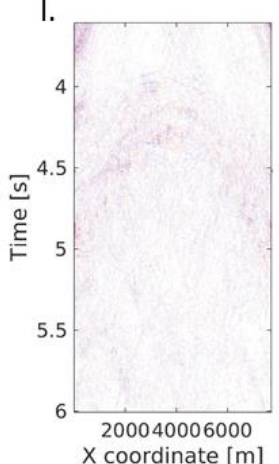

Figure 4 Separation of six simultaneous sources using phase sequencing. (a) to (f) show each of the six sources, separated from the full lines of combined simultaneous source data shown in Figure $1 \mathrm{~g} . \mathrm{g}$ ) to (I) show the separation errors computed by taking the difference of the directly modelled non-simultaneous data, and the separated data in (a) to (f). 